\title{
Diffraction-Enhanced Imaging
}

\author{
Dean M. Connor $\cdot$ Zhong Zhong
}

Published online: 17 May 2014

(C) Springer Science+Business Media New York 2014

\begin{abstract}
Diffraction-enhanced imaging (DEI), sometimes referred to as analyzer-based imaging, is an emerging phase contrast X-ray imaging modality that generates exquisite soft tissue contrast at a vastly reduced absorbed radiation dose relative to absorption-based radiography for imaging applications, which include mammography and assessment of cartilage and lung damage. In this review, we will summarize recent advances in the field of DEI. These include assessment of liver fibrosis, evaluation of the orientation and degree of anisotropy in bone microarchitecture, and assessment of emphysema. We will summarize the state of the clinical translation of DEI and discuss barriers to the clinical translation of DEI and potential ways to overcome these barriers.
\end{abstract}

Keywords Diffraction-enhanced imaging (DEI) .

Analyzer-based imaging (ABI) $\cdot$ Multiple image radiography (MIR) - Phase contrast imaging · Radiography · $\mathrm{X}$-ray vector radiography $\cdot \mathrm{X}$-ray tensor tomography

\section{Introduction}

Diffraction-enhanced imaging (DEI), also referred to as analyzer-based imaging (ABI), is an X-ray-based phase

This article is part of topical collection on New imaging technologies.

\section{M. Connor $(\bowtie)$}

Department of Radiology and Radiological Science, Medical University of South Carolina, 96 Jonathan Lucas St, MSC323, Charleston, SC 29425, USA

e-mail: connord@musc.edu

\section{Z. Zhong}

National Synchrotron Light Source, Brookhaven National

Laboratory, PO Box 5000, Upton, NY 11973, USA

e-mail: zhong@bnl.gov contrast imaging (PCI) system that holds promise as a clinical imaging modality because it can visualize soft tissue features at a vastly reduced radiation dose relative to conventional radiography, and because it can reveal microstructural information that cannot be assessed with conventional radiography.

DEI was independently developed by Chapman [1] and Ingal [2] in the mid-1990s. In DEI, a large, perfect analyzer crystal both converts changes in the angle of a transmitted $\mathrm{X}$-ray beam into intensity changes in an image and removes all scattered photons from the X-ray beam. Figure 1 is a simplified schematic of a DEI system. While DEI can vastly enhance the contrast of soft tissue features and can reveal details of microstructure, it comes at the cost of a requirement for a monochromatic X-ray beam. In this review, we will detail the potential clinical utility of DEI and will provide evidence that the flux restrictions imposed by the monochromatic-requirement can be overcome.

Several different analyzer-based approaches have been developed to extract refraction information. These approaches include a single-image approach [2, 3], a single-refraction-focused two-image approach [1], a multiplerefraction-focused two-image approach [4], and a manyimage approach $[5,6]$. These approaches are referred to as: phase dispersion introscopy, diffraction-enhanced X-ray imaging, DEI, ABI, and multiple image radiography. Throughout this article, we will refer to this collective set of approaches as DEI.

In this review article, we summarize the most influential DEI studies since its inception and shed light on the most recent findings in the field of DEI, and, where appropriate, other applicable PCI findings. Throughout, our focus will be on significant findings that are relevant to clinicians. We have included an Appendix in which we present a primer on the underlying concepts of DEI contrast. 
Fig. 1 Simplified diagram of a DEI system. The slot beam from the DEI system is scanned through the patient to produce an image

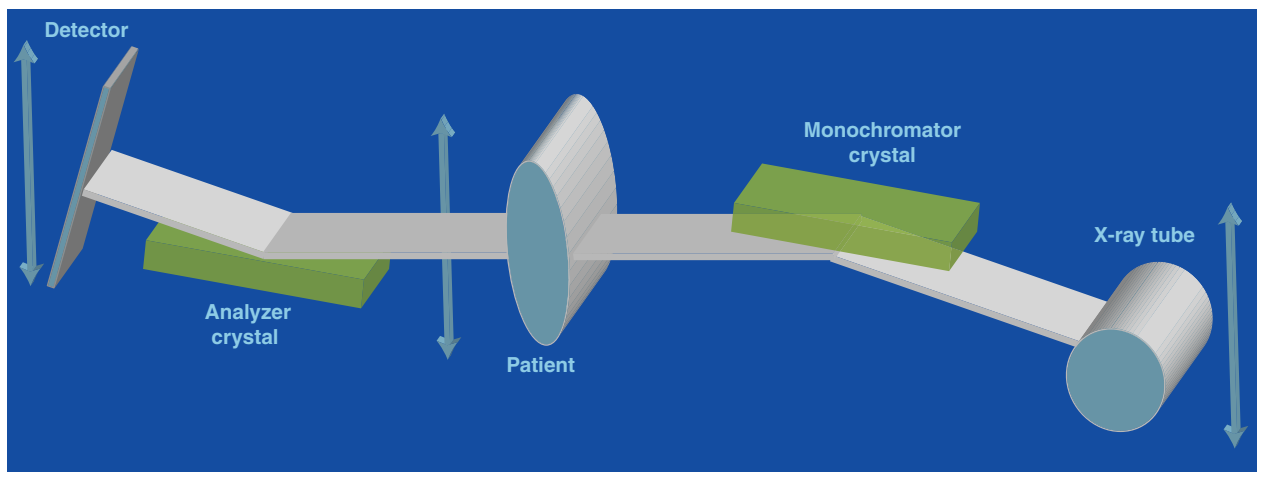

\section{Foundational DEI Studies}

In this section, we will summarize the most important foundational studies in the field of DEI.

\section{Proof-of-Principle DEI Studies}

The following studies laid the foundation of our understanding of the physical mechanisms and limitations of DEI contrast. Chapman et al. [1,3] and Ingal et al. [2] showed that an analyzer crystal could be used to extract refraction information that is carried in an X-ray beam. Dilmanian et al. [7] showed that DEI can be used in a computed tomography mode and that the DEI-CT data could be reconstructed using conventional filtered-backprojection (FBP) algorithms. Oltulu et al. [5] and Wernick et al. [6] showed that the absorption, single-refraction, and multiple-refraction signals can be separated and that CT image sets for each signal can be independently reconstructed with FBP [8]. Kitchen et al. [9] and Khelashvili et al. [10] showed that the origin of the angular spreading of the X-ray beam was multiple-refractions of the beam. Of utmost importance to the clinical translation of DEI, Parham et al. [11••] and Nesch et al. [12] have shown that DEI contrast is maintained when an X-ray tube source is used, and Deimoz et al. have demonstrated that DEI has enhanced signal-to-noise ratio (SNR) compared to gratingbased PCI when imaging at higher X-ray energies [13].

\section{Mammography}

DEI's enhanced soft tissue contrast is of particular importance in mammography. The contrast of spiculations from cancerous breast lesions is greatly enhanced in DEI both with planar and CT imaging [14-16]. Because of its decreased dependence upon X-ray absorption, DEI yields diagnostically useful breast images with a $25 \%$ reduction in breast compression [17]. For calcifications in breast tissue, DEI has five times greater contrast-to-noise ratio than conventional radiography [18]. Thus, DEI can

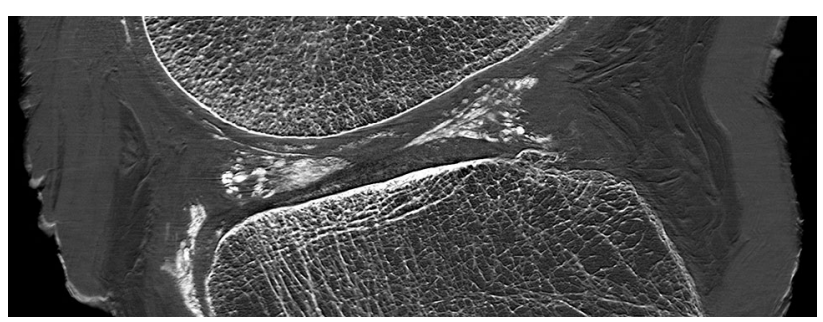

Fig. 2 DEI-CT slice image from an intact cadaveric right knee joint. Images acquired at the half-reflectivity point ( 6.5 microradians) for the $51 \mathrm{keV}, 111$ reflection. Calcinosis can be found in menisci and articular cartilage, the surface of articular cartilage is not smooth. Image courtesy of Jun Li from Rush University Medical College [21]

significantly improve image quality in mammography while both reducing absorbed radiation dose and increasing patient comfort [15].

\section{Joint Imaging}

DEI's enhanced soft tissue contrast is also of particular importance for the imaging of soft tissue within joints. DEI images obtained with X-ray tube sources do not differ significantly from visual inspection (only possible with surgery or post-mortem) in their ability to grade cartilage damage [19, 20]. In CT mode, DEI can visualize both bone and soft tissue with high contrast in an intact cadaveric human knee joint [21] using an absorbed radiation dose equal to that of a clinical image (Fig. 2). Synchrotronbased, very high spatial resolution DEI images can visualize the organization of collagen fibrils in cartilage specimens [22-24] and assess damage to the collagen in cartilage [23]. DEI can also be used to visualize the gap region surrounding bone implants [25-27].

\section{Lung Imaging}

DEI exhibits a unique contrast in the lungs [28, 29]; because of DEI's pronounced multiple-refraction contrast, the lungs appear to be "more absorbing" than bone. To be 

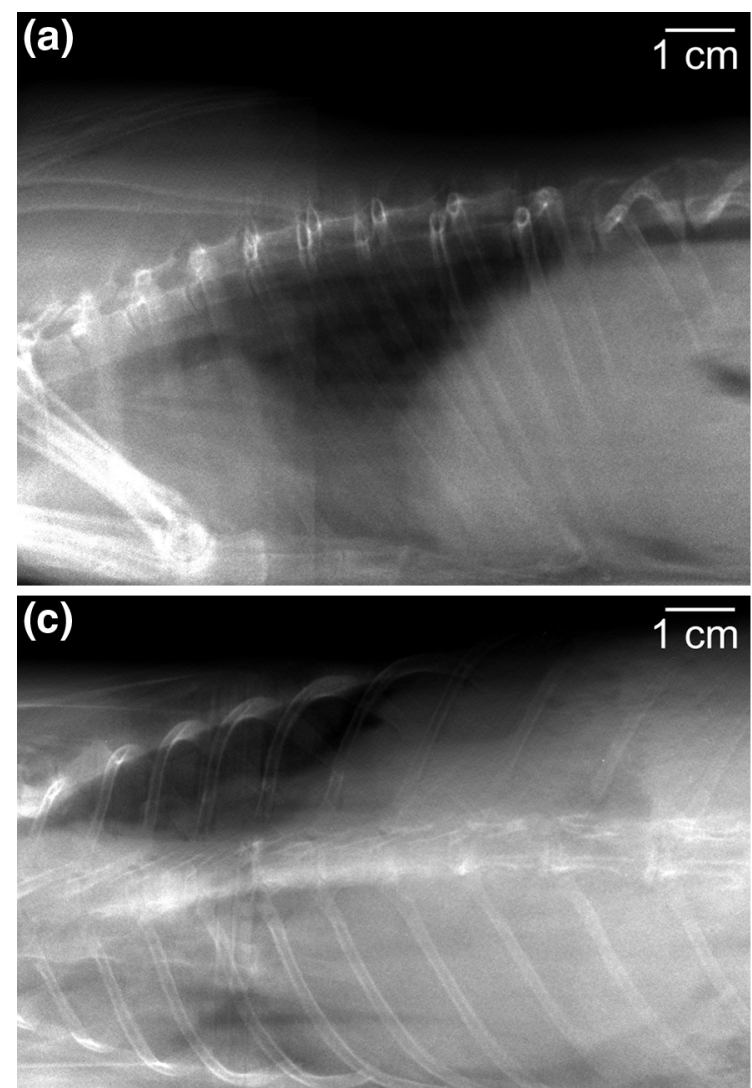

Fig. 3 In vivo chest images from a free-breathing rabbit acquired at the National Synchrotron Light Source. a and c, radiograph images taken along the lateral and anteroposterior (AP) views respectively. b and d, dose-matched DEI peak images. All images were taken at a

clear, this "absorption" is not true absorption (i.e., it is not contributing to the total absorbed dose), but a rejection of X-rays that do not meet the narrow window of angular acceptance of the analyzer crystal. DEI can depict more clearly than conventional radiography the full extent of the lung [29] (Fig. 3) and regions of atelectasis [30].

\section{Recent Advances in DEI}

In the section that follows we will review the most recent literature in the field of DEI. Where applicable, research from other PCI modalities will be summarized as well.

\section{X-ray Vector Radiography}

$\mathrm{X}$-ray vector radiography (XVR) is an emerging field in PCI in which directional information is measured with DEI or grating-based PCI. In XVR, the directional sensitivity of DEI or grating-based PCI is used to generate planar images of the average angular orientation and the variation in the
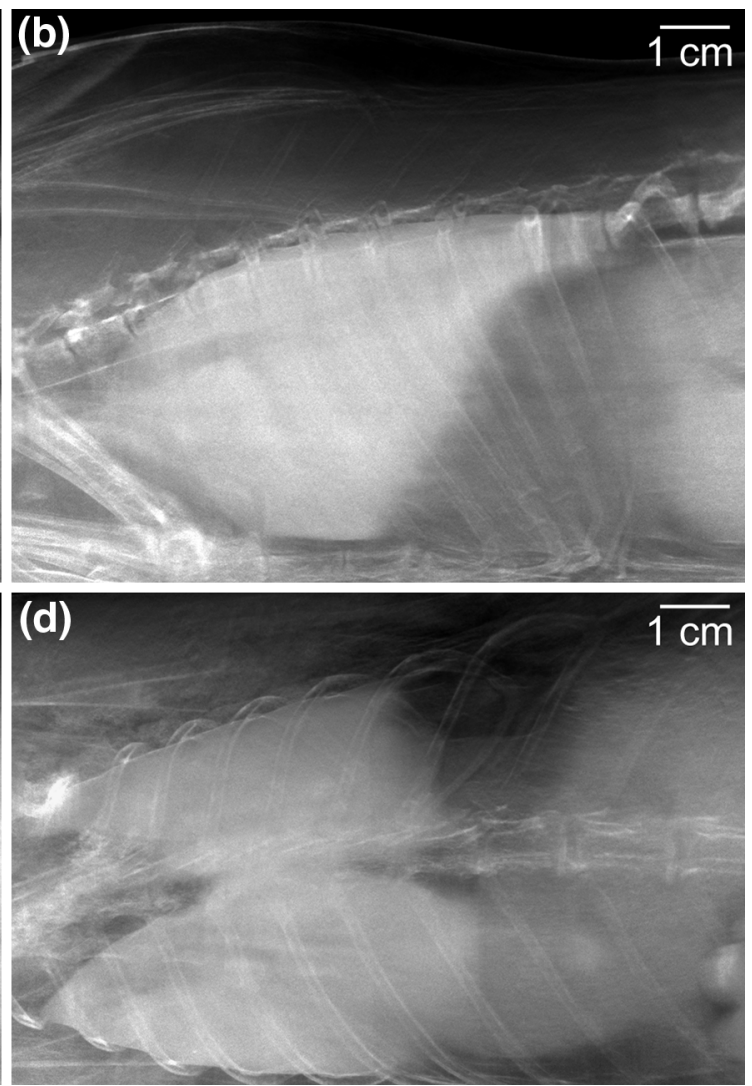

monochromatic X-ray energy of $60 \mathrm{keV}$ and a surface dose of $5 \mu \mathrm{Gy}$. The reversal of contrast in the lungs is due to DEI's multiple refraction contrast

orientation (i.e., the degree of anisotropy) of refracting structures or microstructures along the X-ray beam path [31] through the patient. XVR answers two questions: what is the preferred orientation of microstructures along an X-ray beam path? Also, how strongly aligned are the microstructures?

Malecki et al. [32] have shown that XVR can be extended to a three-dimensional mapping of the angular orientation of the microstructure with an approach called X-ray tensor tomography (XTT). XTT is somewhat analogous to diffusion tensor imaging in MRI. Each voxel in the XTT data set contains discrete information about the attenuation, index of refraction and average angular orientation and the degree of anisotropy in the refracting microstructures within the corresponding volume of the patient.

Bone Microarchitecture

XVR can be used to assess bone microarchitecture. Schaff et al. [33••] (Fig. 4) and Connor et al. [34] have demonstrated that measurements of XVR from grating-based PCI 

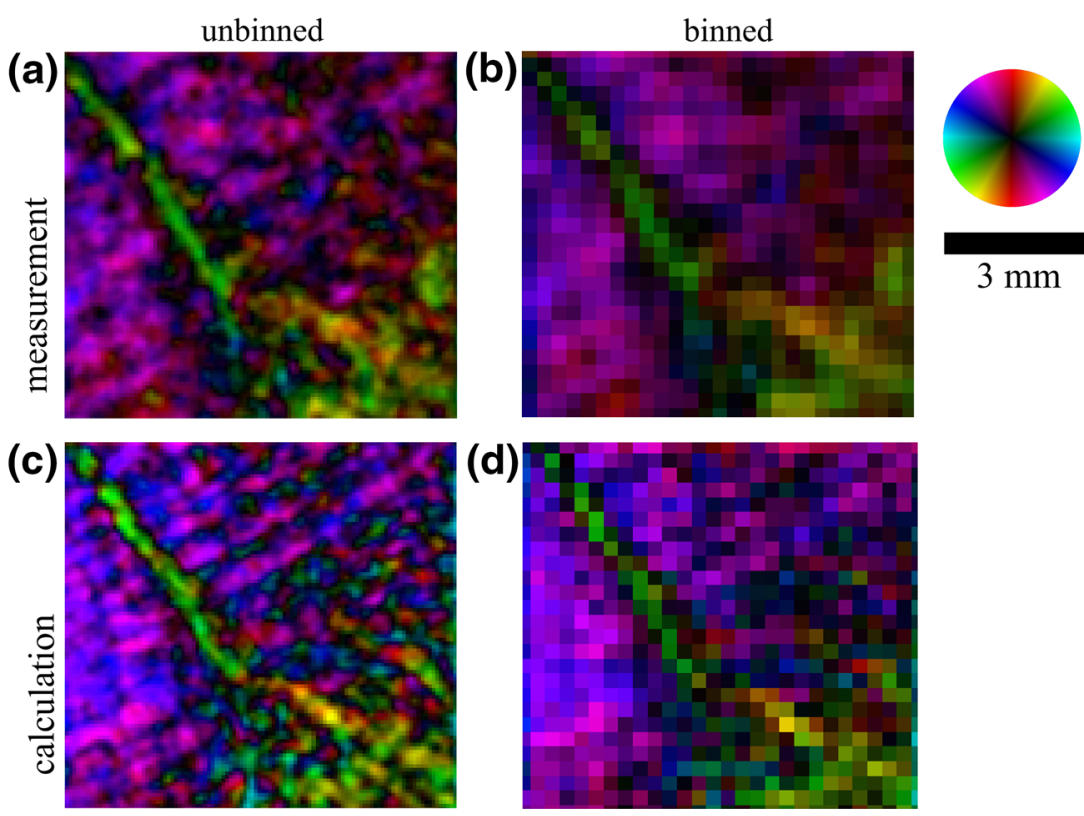

(d)

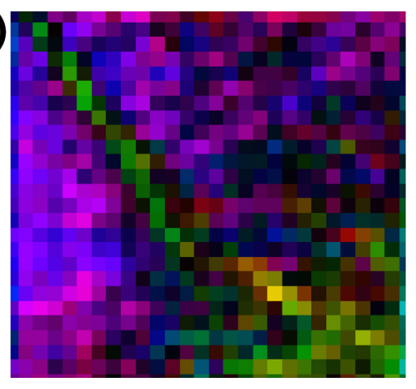

Fig. 4 Schaff XVR image. a XVR measurements of trabecular femoral bone at a detector pixel size of $127 \mu \mathrm{m}^{2}$. The direction of the strongest scattering signal is represented in the color scale given by the color wheel. The brightness of the color corresponds to the anisotropy of the signal, i.e., it shows how strongly oriented the scattering signal is. $\mathbf{b}$ The same data set rebinned by a factor of four is

shown, demonstrating that the XVR information can be retrieved at resolutions much coarser than the underlying trabecular microstructure. $\mathbf{c}$ and $\mathbf{d}$ Corresponding results of a numerical analysis based on highly resolved micro-CT data. This analysis has shown that the recorded XVR signal is formed by the trabecular microstructure. Images courtesy of Florian Schaff and Franz Pfeiffer [33*•]

and DEI, respectively, can be used to measure anisotropy and orientation of bone microarchitecture. This is a significant finding because accurate measures for microarchitectural anisotropy and orientation were previously only possible with either ex vivo high-resolution micro-CT or in vivo at peripheral bone locations, and not at locations where fractures are the most harmful (e.g., hip and vertebrae). Since bone fragility diseases (e.g., osteoporosis) often involve microarchitectural deterioration that leads to fracture, this new measure of the orientation and anisotropy in bone microarchitecture could prove to be a key to predicting fracture.

\section{Contrast Agents for DEI}

While attenuation-based contrast agents will work in DEI and other PCI systems, efforts are underway to develop and test contrast agents that take advantage of the DEI's refraction sensitivity. In order to take full advantage of the refraction contrast of DEI, an ideal DEI contrast agent would have a very low electron density (a very high electron density would also work but that would result in an increase in absorbed radiation dose). Therefore, air is the ideal contrast agent for DEI. Arfelli et al. [35] and Velroyen et al. [36] have shown that microbubbles can be used to enhance contrast in both DEI and grating-based

PCI. Millard et al., have quantified the angular distribution of the X-ray beam as a function of the concentration of microbubbles [37]. Lundström et al. [38] have shown that arterial $\mathrm{CO}_{2}$ can be used to enhance the refraction contrast of both blood vessels and microvessels.

\section{Liver Imaging}

Zhang et al. [39] have shown pronounced phase contrast in the vasculature of a healthy mouse liver that diminishes with increased liver fibrosis. This is somewhat counterintuitive, since we would expect to see fibrosis lead to an enhanced multiple-refraction signal. But there is a significant decrease in the liver vasculature caused by accumulated extracellular matrix proteins in the liver that significantly overpowers the fiber-based enhancement of the refraction signal. Duan et al. [40] have extended this analysis showing that DEI-CT can assess morphological changes in liver microvasculature that result from fibrosis. Hu et al. [41•] have used these morphological measures to show a 1.4-fold and twofold reduction in the number density of blood vessels in fibrotic and cirrhotic rats, respectively. The high-resolution DEI-CT approach used in these studies does not directly translate into clinical imaging, but this approach can be used as a gold standard against which multiple-refraction and XVR images can be 


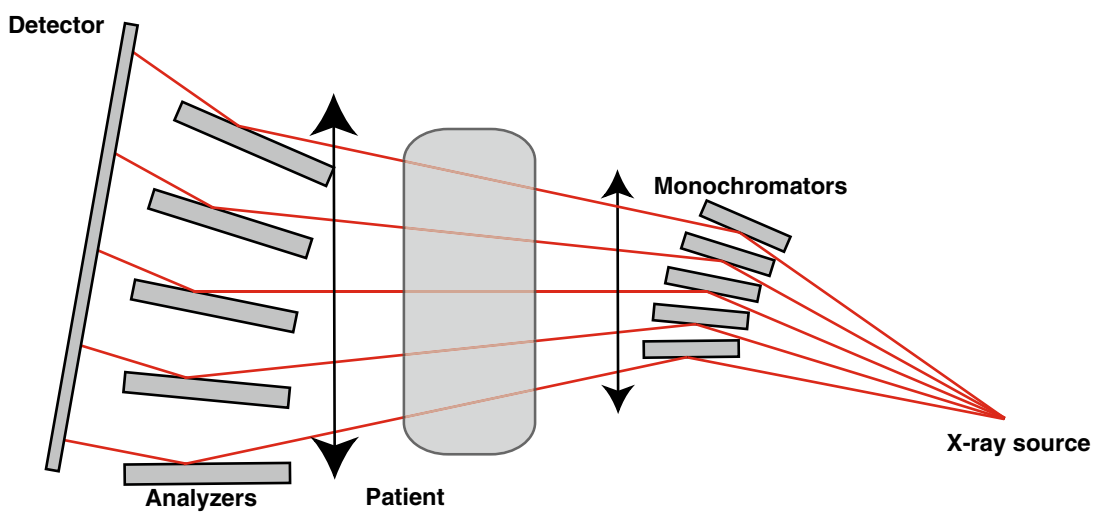

Fig. 5 Schematic of a proposed multiple-beam array DEI system. In this configuration, each monochromator/analyzer crystal pair is an independent DEI system

compared. The high-resolution DEI-CT can help to assess the effectiveness of the lower resolution planar DEI approaches for differentiating between healthy and diseased liver.

\section{Pulmonary imaging}

As was shown in Kitchen et al., and Connor et al.'s work with DEI of lungs and pulmonary injury [29, 30], Schwab et al. [42] have shown that the dark-field image of grating-based PCI has significantly enhanced contrast relative to an attenuation image. Schleede et al. [43] have shown that the darkfield images from grating-based PCI contain sufficient contrast to distinguish between emphysemic and healthy lungs in a mouse model of emphysema. Meinel et al. [44•] have developed a "normalized scatter" image (where the darkfield image is normalized to the attenuation image) and have shown that the normalized scatter image is more sensitive to emphysema in the lungs than an attenuation image.

\section{Clinical Translation of DEI}

Two competing approaches to X-ray tube-based DEI have been proposed and pre-clinical prototypes of these systems have been reported by Parham et al. [11•0] and Nesch et al. [12]. Parham's DEI system utilizes symmetric crystals to generate a slot X-ray beam DEI system while Nesch's system utilizes asymmetric crystals to produce an area X-ray beam. An advantage of Parham's system is that it more readily translates to higher energy, even as high energy as the tungsten $K \alpha 1$ and $K \alpha 2$ emission energies of 59.318 and $57.982 \mathrm{keV}$, respectively; the high energy of DEI is a compelling advantage because DEI's SNR is weakly coupled to energy ( $\mathrm{SNR} \propto 1 / \mathrm{E})[13]$ and because the enhanced transmission of X-rays at higher energies eases the flux constraints of DEI. A disadvantage of Parham's approach is that it makes inefficient usage of the full
X-ray beam. An advantage of Nesch's approach is that it makes more efficient use of the full X-ray beam, and it does not require translation of the X-ray beam to generate an image. A disadvantage of Nesch's approach is that it does not translate well to higher energies, as the size of the crystal optics have to be large relative to the size of the imaging field at higher energies. In our opinion, Parham's slot-beam DEI approach is the more likely approach to succeed in a clinical setting, while Nesch's approach is more likely to succeed as a small animal planar and CT imaging system.

Because DEI requires a monochromatic X-ray beam, flux and imaging time are the largest obstacles to the clinical translation of DEI. Parham et al., measured the flux and imaging time for the tungsten anode X-ray tube-based DEI system $[11 \bullet \cdot]$. While the image acquisition time on that proof-of-principle system was very long, Connor et al. [45] have demonstrated that a higher power, rotating anode $\mathrm{X}$-ray tube source can be utilized in a DEI system and reduce the imaging time significantly, but the image acquisition times are currently on the order of tens of minutes to hours.

Fundamentally, the major advancement that will need to be made to translate DEI into the clinic is that the full, divergent X-ray beam must be more efficiently used in order to reduce imaging time down to a time that coincides with the maximum time an X-ray source can be operated at high-power. Single-beam DEI systems make inefficient use of the divergent $\mathrm{X}$-ray beam generated by $\mathrm{X}$-ray tube sources. It is our opinion that the best solution to this problem is to build a multiple-beam array DEI system, where each individual beam is its own DEI system and scans a different part of the image field (Fig. 5). An $n$-beam DEI system will reduce the acquisition time by a factor of $n$.

Rotating anode tube sources can be operated in two modes, a low average power mode and a high power mode. 


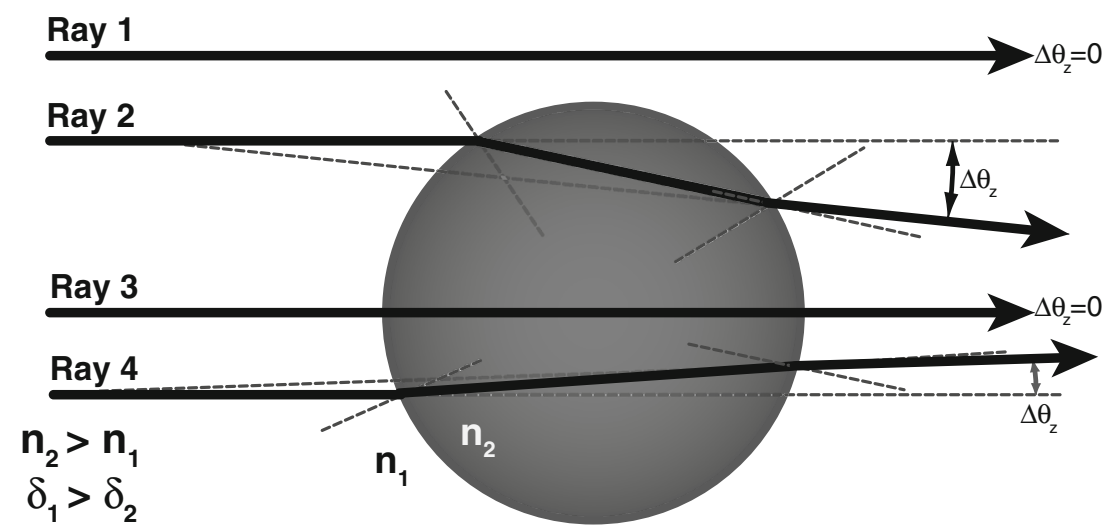

Fig. 6 Refraction from a single object in an X-ray beam. An X-ray beam that intersects the object is not necessarily refracted (Ray 3). The amount of X-ray refraction depends on the angle of incidence on the object; the higher the angle of incidence, the larger the amount of refraction (refraction is greater for Ray 2 than Ray 4)

contrast can be broken down into two major categories: single refraction contrast, where the refraction signal is dominated by a single refracting structure, and multiple refraction contrast, where the refraction signal is dominated by the net effect of many refracting structures. Multiple refraction contrast is sometimes referred to as ultra-small angle X-ray scattering or extinction contrast in DEI papers or dark-field imaging in grating-based PCI papers, but the physical mechanism of contrast is multiple refractions of the X-ray beam in all cases.

Both types of DEI refract contrast are driven by two parameters, the amount the X-ray beam has been refracted and the angular position of the analyzer crystal. Our discussion of DEI's refraction contrast will begin with a description of X-ray refraction in both the single-refraction and multiple-refraction cases and continue with a description of the analyzer crystal's role in DEI contrast.

\section{Single Refracting Object}

To understand how an X-ray beam interacts with a single refracting object, we will follow a light ray as it passes through an object, in this case a cylinder (Fig. 6). As the ray of light (in our case an X-ray beam) passes between a medium of one index of refraction into a second medium of a different index of refraction, the light will be deflected away from its straight-line path (Rays 2 and 4, Fig. 6); in other words, it will be refracted. The light will be refracted a second time as it passes back into the first medium. If we trace the ray of light through the object, we can determine the total change in the angle of the ray of light from its initial path, $\Delta \theta$, the total refraction. Refraction has a directional component; note that Ray 4 is deflected upward while Ray 2 is deflected downward and Ray 3 is undeflected (Fig. 6). If we imagine the object in Fig. 6 is a 


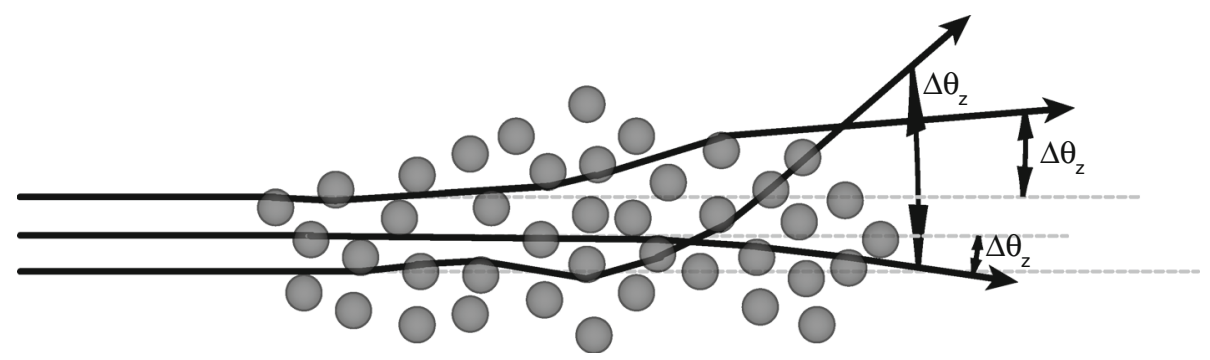

Fig. 7 Refraction from multiple objects in an X-ray beam. X-rays travelling in the same direction as that enter the multiply-refracting object will exit the object at a range of angles with respect to their straight line path

sphere, then the angular deflection can be some combination of upward/downward and into-/out-of-the-page, and we can break $\Delta \theta$ down into its upward/downward component, $\Delta \theta_{\mathrm{z}}$, and its into-/out-of-the-page component, $\Delta \theta_{\mathrm{y}}$.

Two factors affect the magnitude of the change in angle of the X-ray beam: the difference in index of refraction between the two media and the angle between the X-ray beam and the interface(s) between the media. The index of refraction is $n=1+\delta+i \beta$, where $\delta$ is the real part of the index of refraction that effects the angular deflection of the $X$-ray beam and $\beta$ is the imaginary part of the index of refraction that effects the attenuation of the X-ray beam. The real part of the index of refraction, $\delta$, varies as a function of the energy of the light $\left(\delta \propto 1 / E^{2}\right)$ and as a function of the electron density of the material $\left(\delta \propto \rho_{e}\right)$ in most cases $\rho_{e}$ closely matches the physical density, $\rho$, of the material). For clinically relevant X-ray energies and for most biological tissues, $\delta$ is very small, as are differences in $\delta$ between adjacent media, $\Delta \delta$; these small differences result in very small changes in the angle of the deflected beam-on the order of $10^{-7}$ radians, which is the angle subtended by $100 \mathrm{~nm}$ at $1 \mathrm{~m}$. Over the length-scale of a clinical imaging system (object-to-detector distance of much less than $1 \mathrm{~m}$ ), the angular displacement occurs well within the pixel $(100 \mathrm{~nm} \ll 75 \mu \mathrm{m}$ pixel pitch in a flatpanel radiography system).

The angle between the X-ray beam and the interface also affects the amount of refraction. If $\alpha$ is the angle between the surface normal (perpendicular to the surface) and the X-ray beam, then $\Delta \theta \propto \tan \alpha$. The closer the $\mathrm{X}$-ray beam is to perpendicular to the interface, the smaller the amount of refraction (Ray 3 in Fig. 6). Conversely, the amount of refraction increases as the $\mathrm{X}$-ray beam becomes closer to parallel to the interface (Rays 2 and 4 in Fig. 6).

In summary, refraction from a single object in the imaging field looks like a steering of the X-ray beam and the total magnitude of the refraction of the X-ray beam is proportional to the difference in the electron densities between the two media, inversely proportional to the X-ray energy squared, and proportional to $\tan \alpha\left(\Delta \theta \propto \Delta \rho_{e^{*}} \tan \alpha / E^{2}\right)$.

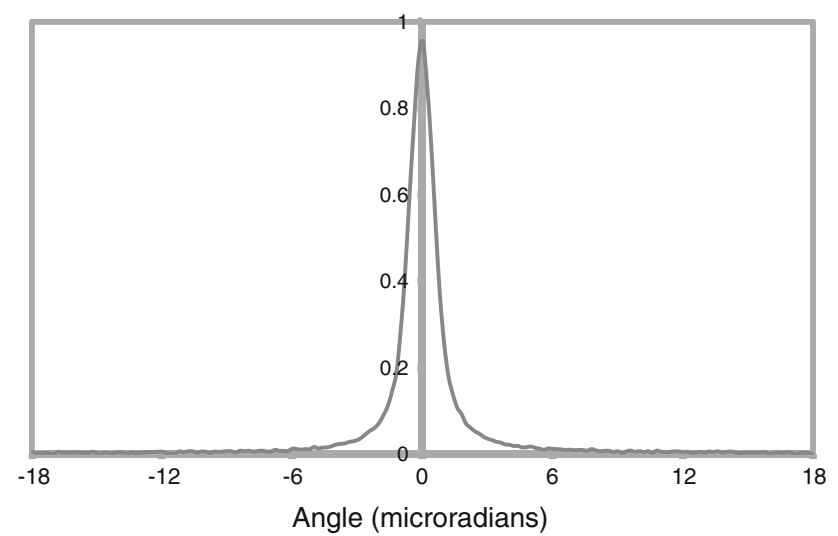

Fig. 8 Example reflectivity profile from a DEI system taken at about $60 \mathrm{keV}$ with the silicon 333 reflection. Note that the maximum slope of the reflectivity profile is approximately at the half-maximum point on the reflectivity profile and that the reflectivity profile is approximately linear over the range immediately surrounding the halfmaximum point

\section{Multiple Refracting Objects}

The case of multiple refracting objects in the X-ray beam is just an extension of the single refracting object case for multiple refracting objects in the beam. If multiple refracting objects are placed in the X-ray beam path (Fig. 7), then the X-ray beam will be refracted many times. While each individual refracting object can be thought of as steering the X-ray beam, the net effect of many refracting objects is diffusing the angle of the X-ray beam.

The amount that the X-ray beam diffuses is related to the amount of refraction from each individual object (see "Single refraction object" section above) and the number of refracting objects along the beam path. The more refracting objects along the beam path the more the X-ray beam will be diffused.

\section{Role of the Analyzer Crystal in DEI Contrast}

The analyzer crystal can be thought of as the gatekeeper that controls access to the imaging detector. X-rays that 

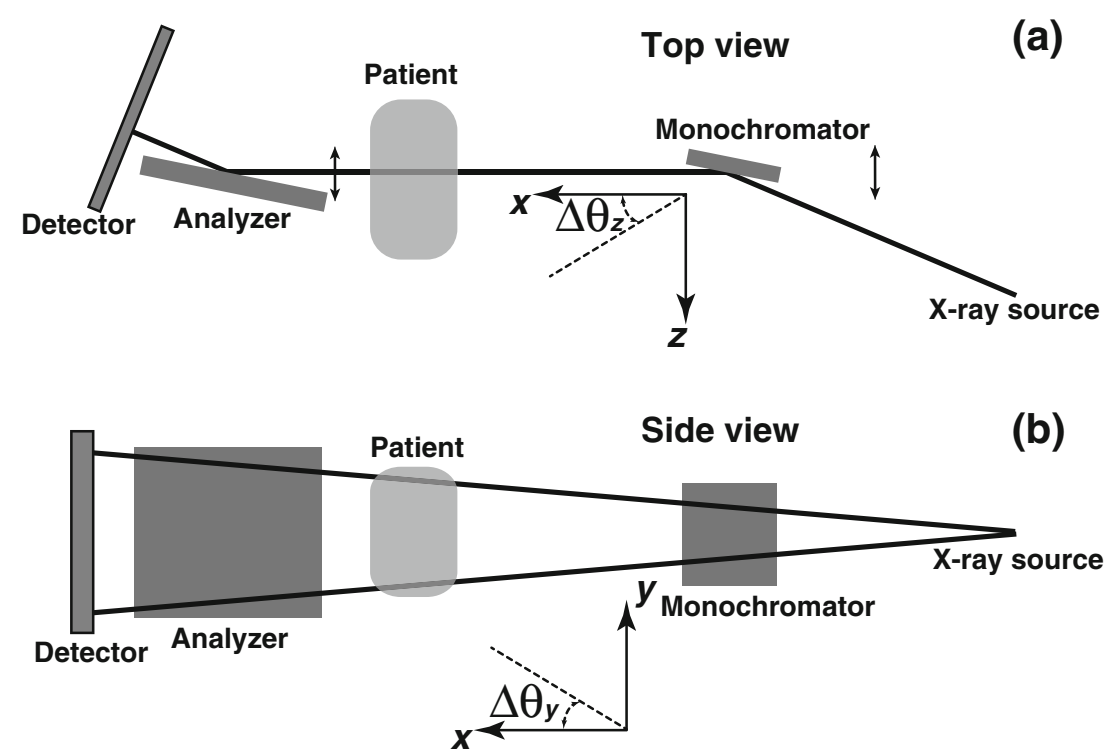

Fig. 9 a Top view and $\mathbf{b}$ side view of a DEI system. DEI is sensitive to changes in the angle $\Delta \theta_{\mathrm{z}}$ and insensitive to changes in the angle $\Delta \theta_{\mathrm{y}}$
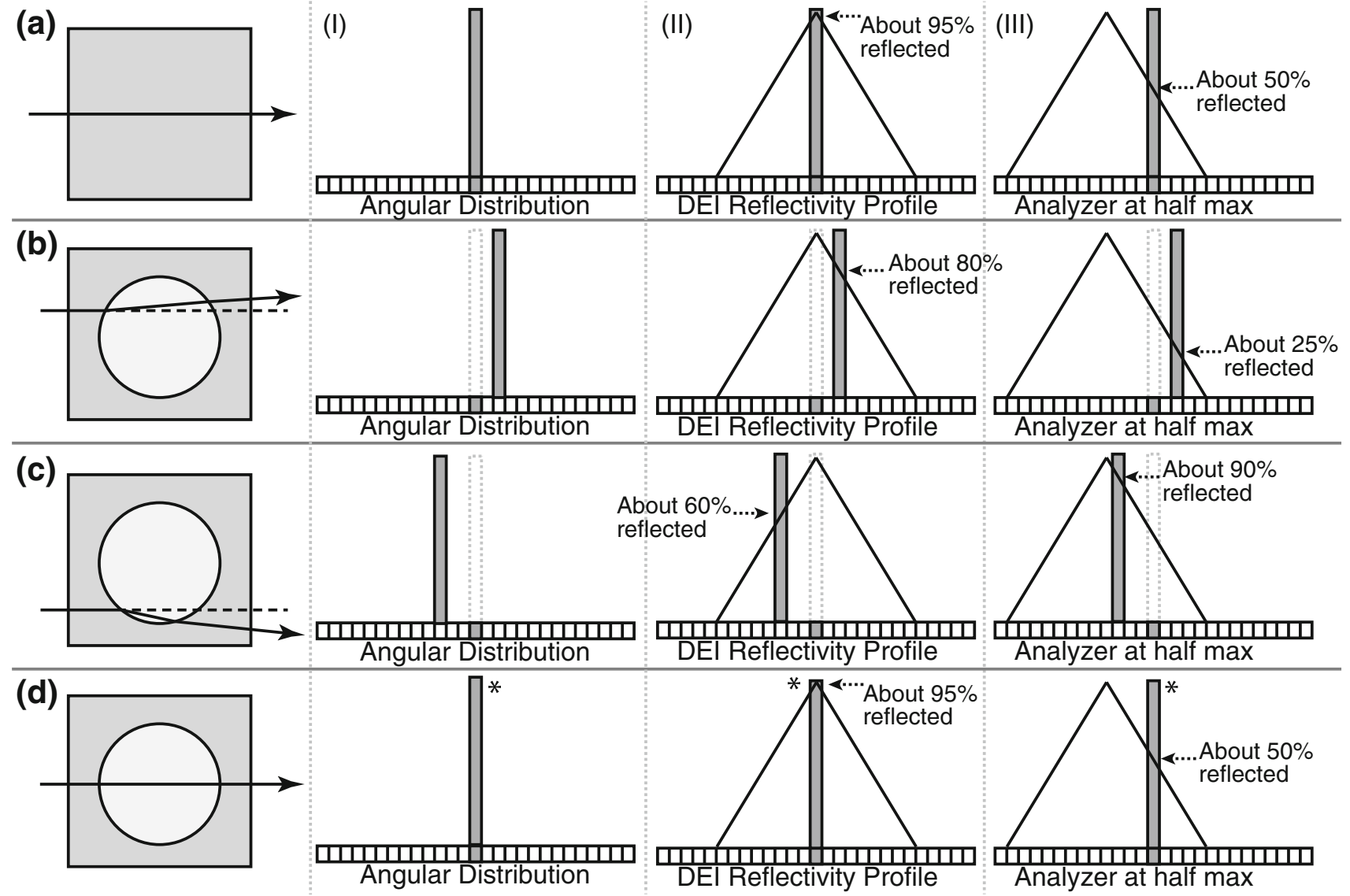

Fig. 10 Rows a-d represent 4 different cases of refraction from a single object. Case $\mathbf{b}$ is a small upward (positive) refraction, while $\mathbf{c}$ is a larger, downward (negative) refraction. Column (I) depicts the change in the angular distribution. Column (II) depicts the reflectivity
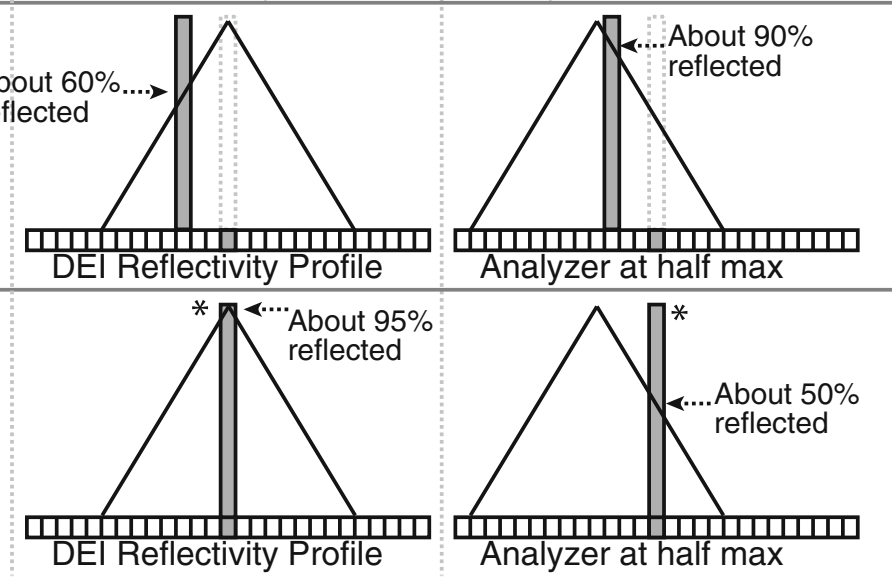

for when the analyzer crystal is aligned to undeflected X-ray beam. Column (III) depicts the reflectivity for when the analyzer crystal is aligned to reflect half of the undeflected X-ray beam 
Fig. 11 Rows a-d represent increasing numbers of refracting objects in the X-ray beam path. Column (I) depicts the change in the angular distribution. In each case $\mathbf{a}-\mathbf{d}$, the total area under the angular distribution curve is the same (X-rays are spread out in angle, but not absorbed). Column (II) depicts the new reflectivity profile (solid line) relative to the original reflectivity profile (dotted line)
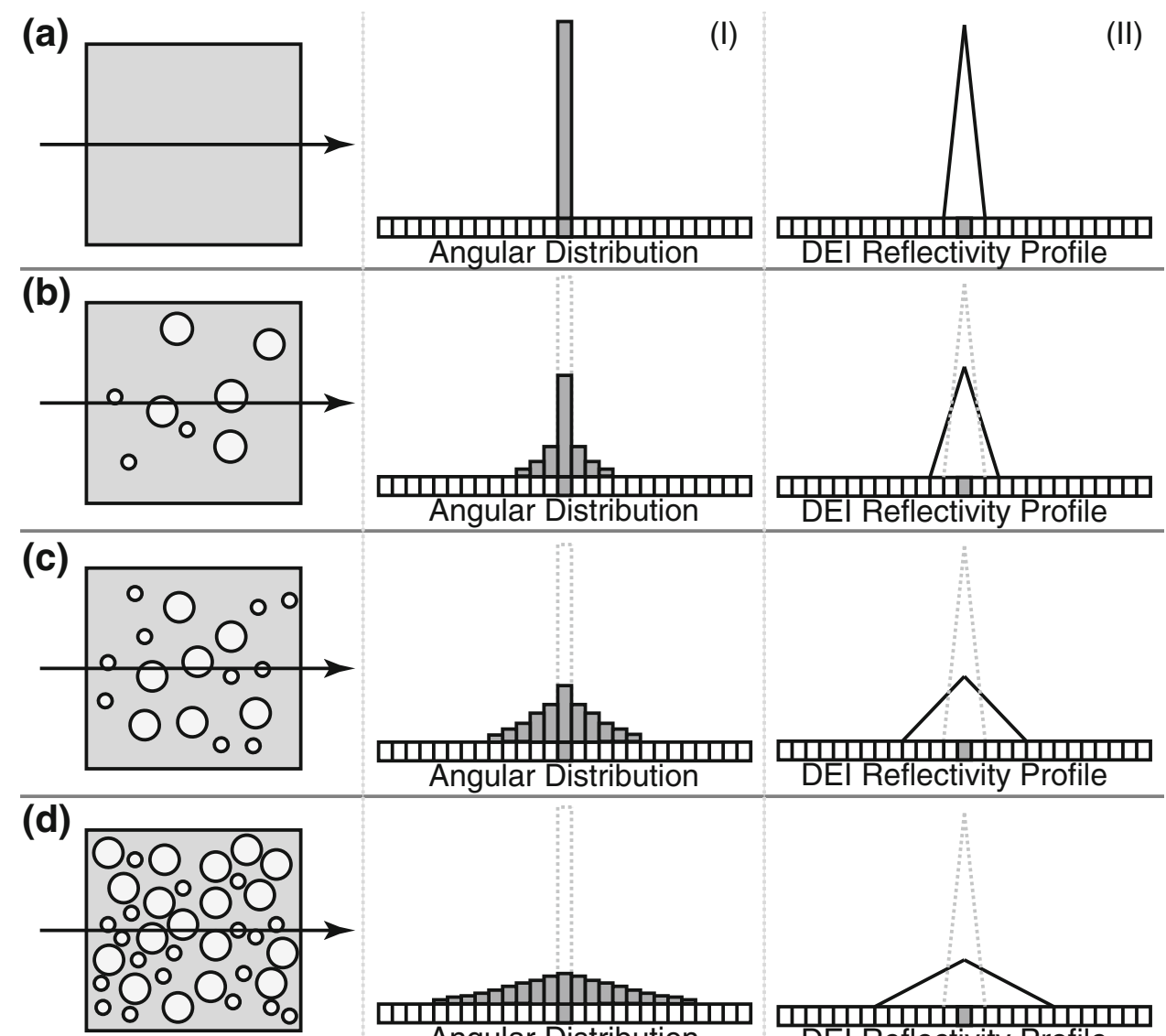

intersect the analyzer crystal will be "analyzed" to determine if they are at the proper angle and energy (i.e., do they meet the diffraction conditions for the analyzer crystal). $\mathrm{X}$-rays that have both the proper angle and energy will be allowed to pass (reflected) by the analyzer crystal, with a small tax-about $5 \%$ of the X-ray beam that meets the diffraction conditions will not be reflected. X-rays that are very close to the proper energy and angle will still be partially reflected, but the tax becomes higher; the tax very quickly approaches $100 \%$, typically within about a few microradians of the proper angle. We can plot the percentage of X-rays that are allowed to pass by the analyzer crystal as a function of how much they deviate from the proper angle or energy; this plot is referred to as the reflectivity profile, or the rocking curve, of the DEI system (Fig. 8). The width of the reflectivity profile decreases inversely with energy (width $\propto 1 / \mathrm{E}$ ). As a result, the slope of the straight region of the reflectivity profile (at about the half-maximum points) increases directly with energy (slope $\propto E$ ). The slope of the reflectivity profile curve is steepest in the near-linear region around its half-width points.

The analyzer has a direction-specific angular sensitivity. The DEI optical system is highly sensitive to changes in the angle of the X-ray beam in the $z$-direction, $\Delta \theta_{z}$ (Fig. 9).
The DEI system is insensitive to changes in the X-ray beam along the $y$-direction, $\Delta \theta_{y}$.

\section{Single Refraction DEI Contrast}

By manipulating the analyzer crystal position, we can create image contrast. We can control the analyzer crystal by slightly tilting the angle away from the proper angle (for diffraction); this will slightly change the proper alignment angle of the X-ray beam. For example, we can tilt the analyzer to a position where $50 \%$ of the undeflected X-rays are reflected by the analyzer crystal (Fig. 10a, column III). If we then place a single refracting object in the X-ray beam, it can steer the X-ray beam away from (Fig. 10b, column III) or towards (Fig. 10c, column III) the proper angle for full reflection. The analyzer crystal will reflect more than $50 \%$ of the X-rays that are steered towards the proper angle of the imaging system and fewer than $50 \%$ of the X-rays that are steered away from the proper angle of the imaging system. This steering of X-rays towards or away from the proper angle (maximum reflection) is the essence of image contrast in a DEI system.

In the single-refracting object case, the amount of contrast in a DEI system is dictated by the total change in the 
angle of the X-ray beam (the amount of refraction) and the slope of the reflectivity profile. Increasing the energy in the $\mathrm{X}$-ray beam will reduce the total change in angle $\left(\Delta \theta_{z} \propto 1 /\right.$ $\left.E^{2}\right)$ and will increase the slope of the reflectivity profile (slope $\propto E$ ). As a result, DEI contrast only decreases as $1 /$ $E$; doubling the energy of the X-ray beam only results in a two-fold decrease in image contrast.

\section{Multiple Refraction DEI Contrast}

In the multiple-refracting objects case, the $\mathrm{X}$-ray beam is angularly diffused (Fig. 11, Column I). The total number of $\mathrm{X}$-rays is unchanged, but the X-rays are distributed in angle. Now if we align the analyzer crystal to the proper angle, some of the X-rays will meet the conditions for nearly $100 \%$ reflection, but many X-rays will be misaligned from the proper angle and will pay a higher tax for reflection. The more the X-ray beam is angularly diffused, the higher the average tax will be. The "higher tax" will look like absorption in the final image, but no absorption is required to generate this contrast.

If we measure the reflectivity profile in the multiplerefracting objects case, we will find that it is broadened (the measured reflectivity profile is the convolution of the intrinsic reflectivity profile with the Gaussian angular spreading of the multiple-refracting objects). The reflectivity profile width increases in proportion to the square root of the number of times the X-ray beam has been refracted (width $\propto n^{1 / 2}{ }^{1 / 2}$; Fig. 11, Column II) and to the difference in density between the refracting objects and the bulk tissue matrix $\left(\Delta \theta \propto \Delta \rho_{e}\right)$. Therefore, multiple refraction contrast is greatest for objects with many refracting features that are each drastically different in density from the bulk tissue matrix such as air-filled alveoli in lung and the spongy framework of trabecular bone.

\section{Compliance with Ethics Guidelines}

Conflict of Interest Dr. Dean Connor and Dr. Zhong Zhong are cofounders of and part owners of NextRay, Inc., a start-up company that is developing a commercial DEI system.

Human and Animal Rights and Informed Consent This article does not contain any studies with human or animal subjects performed by any of the authors.

\section{References}

Recently published papers of particular interest have been highlighted as:

- Of importance

•- Of major importance
1. Chapman D, Thomlinson W, Johnston RE, Washburn D, Pisano E, Gmur N, et al. Diffraction enhanced X-ray imaging. Phys Med Biol. 1997;42(11):2015-25.

2. Ingal VN, Beliaevskaya EA. Phase dispersion introscopy. Surf Invest. 1997;12:441-50.

3. Chapman D, Thomlinson W, Arfelli F, Gmür N, Zhong Z, Menk $\mathrm{R}$, et al. Mammography imaging studies using a Laue crystal analyzer. Rev Sci Instrum. 1996;67(9):3360.

4. Rigon L, Besch HJ, Arfelli F, Menk RH, Heitner G, PlothowBesch H. A new DEI algorithm capable of investigating sub-pixel structures. J Phys D-Appl Phys. 2003;36(10A):A107-12.

5. Oltulu O, Zhong Z, Hasnah M, Wernick MN, Chapman D. Extraction of extinction, refraction and absorption properties in diffraction enhanced imaging. J Phys D-Appl Phys. 2003;36(17): 2152-6.

6. Wernick MN, Wirjadi O, Chapman D, Zhong Z, Galatsanos NP, Yang YY, et al. Multiple-image radiography. Phys Med Biol. 2003;48(23):3875-95.

7. Dilmanian FA, Zhong Z, Ren B, Wu XY, Chapman LD, Orion I, et al. Computed tomography of X-ray index of refraction using the diffraction enhanced imaging method. Phys Med Biol. 2000;45(4):933-46.

8. Brankov JG, Wernick MN, Yang Y, Li J, Muehleman C, Zhong $\mathrm{Z}$, et al. A computed tomography implementation of multipleimage radiography. Med Phys. 2006;33(2):278-89.

9. Kitchen MJ, Paganin D, Lewis RA, Yagi N, Uesugi K, Mudie ST. On the origin of speckle in X-ray phase contrast images of lung tissue. Phys Med Biol. 2004;49(18):4335-48.

10. Khelashvili G, Brankov JG, Chapman D, Anastasio MA, Yang Y, Zhong Z, et al. A physical model of multiple-image radiography. Phys Med Biol. 2006;51(2):221-36. doi:10.1088/0031-9155/51/ 2/003.

11. • Parham C, Zhong Z, Connor DM, Chapman LD, Pisano ED. Design and implementation of a compact low-dose diffraction enhanced medical imaging system. Acad Radiol. 2009;16(8): 911-7. doi: 10.1016/j.acra.2009.02.007. In this study, Parham et al., demonstrate the feasibility of an X-ray tube-based diffraction-enhanced imaging system and present an argument for clinical feasibility.

12. Nesch I, Fogarty DP, Tzvetkov T, Reinhart B, Walus AC, Khelashvili G, et al. The design and application of an in-laboratory diffraction-enhanced X-ray imaging instrument. Rev Sci Instrum. 2009;80(9):093702. doi:10.1063/1.3213621.

13. Diemoz PC, Bravin A, Coan P. Theoretical comparison of three $\mathrm{X}$-ray phase-contrast imaging techniques: propagation-based imaging, analyzer-based imaging and grating interferometry. Opt Express. 2012;20(3):2789-805. doi:10.1364/oe.20.002789.

14. Pisano ED, Johnston RE, Chapman D, Geradts J, Iacocca MV, Livasy CA, et al. Human breast cancer specimens: diffractionenhance imaging with histologic correlation-Improved conspicuity of lesion detail compared with digital radiography. Radiology. 2000;214(3):895-901.

15. Keyrilainen J, Fernandez M, Karjalainen-Lindsberg ML, Virkkunen P, Leidenius M, von Smitten K, et al. Toward high-contrast breast CT at low radiation dose. Radiology. 2008;249(1):321-7. doi:10.1148/radiol.2491072129.

16. Kao T, Connor D, Dilmanian FA, Faulconer L, Liu T, Parham C, et al. Characterization of diffraction-enhanced imaging contrast in breast cancer. Phys Med Biol. 2009;54(10):3247-56. doi:10. 1088/0031-9155/54/10/019.

17. Faulconer LS, Parham CA, Connor DM, Kuzmiak C, Koomen M, Lee $\mathrm{Y}$, et al. Effect of breast compression on lesion characteristic visibility with diffraction-enhanced imaging. Acad Radiol. 2010;17(4):433-40. doi:10.1016/j.acra.2009.10.020. 
18. Kiss MZ, Sayers DE, Zhong Z, Parham C, Pisano ED. Improved image contrast of calcifications in breast tissue specimens using diffraction enhanced imaging. Phys Med Biol. 2004;49(15): 3427-39.

19. Muehleman C, Fogarty D, Reinhart B, Tzvetkov T, Li J, Nesch I. In-laboratory diffraction-enhanced X-ray imaging for articular cartilage. Clin Anat. 2010;23(5):530-8. doi:10.1002/ca.20993.

20. Muehleman C, Li J, Connor D, Parham C, Pisano E, Zhong Z. Diffraction-enhanced imaging of musculoskeletal tissues using a conventional X-ray tube. Acad Radiol. 2009;16(8):918-23. doi:10.1016/j.acra.2009.04.006.

21. Li J, Zhong Z, Connor D, Mollenhauer J, Muehleman C. Phasesensitive X-ray imaging of synovial joints. Osteoarthr Cartil. 2009;17(9):1193-6. doi:10.1016/j.joca.2009.03.005.

22. Muehleman C, Majumdar S, Issever AS, Arfelli F, Menk RH, Rigon L, et al. X-ray detection of structural orientation in human articular cartilage. Osteoarthr Cartil. 2004;12(2):97-105.

23. Li J, Jadin K, Masuda K, Sah R, Muehleman C. Characterization of lesions of the talus and description of tram-track lesions. Foot Ankle Int. 2006;27(5):344-55.

24. Majumdar S, Issever AS, Burghardt A, Lotz J, Arfelli F, Rigon L, et al. Diffraction enhanced imaging of articular cartilage and comparison with micro-computed tomography of the underlying bone structure. Eur Radiol. 2004;14(8):1440-8. doi:10.1007/ s00330-004-2355-8.

25. Coan P, Mollenhauer J, Wagner A, Muehleman C, Bravin A. Analyzer-based imaging technique in tomography of cartilage and metal implants: a study at the ESRF. Eur J Radiol. 2008;68(3 Suppl):S41-8. doi:10.1016/j.ejrad.2008.04.036.

26. Wagner A, Sachse A, Keller M, Aurich M, Wetzel WD, Hortschansky $\mathrm{P}$, et al. Qualitative evaluation of titanium implant integration into bone by diffraction enhanced imaging. Phys Med Biol. 2006;51(5):1313-24. doi:10.1088/0031-9155/51/5/019.

27. Connor DM, Sayers D, Sumner DR, Zhong Z. Diffraction enhanced imaging of controlled defects within bone, including bone-metal gaps. Phys Med Biol. 2006;51(12):3283-300. doi:10. 1088/0031-9155/51/12/019.

28. Zhong Z, Thomlinson W, Chapman D, Sayers D. Implementation of diffraction-enhanced imaging experiments: at the NSLS and APS. Nucl Instrum Methods Phys Res A. 2000;450:556-67.

29. Kitchen MJ, Lewis RA, Yagi N, Uesugi K, Paganin D, Hooper $\mathrm{SB}$, et al. Phase contrast X-ray imaging of mice and rabbit lungs: a comparative study. Br J Radiol. 2005;78(935):1018-27. doi:10. 1259/bjr/13024611.

30. Connor DM, Zhong Z, Foda HD, Wiebe S, Parham CA, Dilmanian FA, et al. Diffraction enhanced imaging of a rat model of gastric acid aspiration pneumonitis. Acad Radiol. 2011;18(12): 1515-21. doi:10.1016/j.acra.2011.08.005.

31. Malecki A, Potdevin G, Biernath T, Eggl E, Garcia EG, Baum T, et al. Coherent superposition in grating-based directional darkfield imaging. PLoS One. 2013;8(4):e61268.

32. Malecki A, Potdevin G, Biernath T, Eggl E, Willer K, Lasser T, et al. X-ray tensor tomography. EPL (Europhysics Letters). 2014; 105(3):38002

33. •• Schaff F, Malecki A, Potdevin G, Eggl E, Noël PB, Baum T et al. Correlation of X-ray vector radiography to bone micro- architecture. Sci Rep. 2014;4. doi:10.1038/srep03695. This paper details how the average orientation and the degree of anisotropy in bone microarchitecture can be measured with PCI.

34. Connor DM, Mehrotra M, LaRue AC. Quantification of microarchitectural anisotropy in bone with diffraction enhanced imaging SPIE Medical Imaging: Physics of Medical Imaging 2014. p. 9033-82.

35. Arfelli F, Rigon L, Menk RH. Microbubbles as X-ray scattering contrast agents using analyzer-based imaging. Phys Med Biol. 2010;55(6):1643-58. doi:10.1088/0031-9155/55/6/008.

36. Velroyen A, Bech M, Malecki A, Tapfer A, Yaroshenko A, Ingrisch $\mathrm{M}$, et al. Microbubbles as a scattering contrast agent for grating-based X-ray dark-field imaging. Phys Med Biol. 2013;58(4):N37.

37. Millard T, Endrizzi M, Rigon L, Arfelli F, Menk R, Owen J, et al. Quantification of microbubble concentration through X-ray phase contrast imaging. Appl Phys Lett. 2013;103(11):114105.

38. Lundström U, Larsson DH, Burvall A, Takman PA, Scott L, Brismar $\mathrm{H}$, et al. X-ray phase contrast for $\mathrm{CO} 2$ microangiography. Phys Med Biol. 2012;57(9):2603.

39. Zhang X, Yang X-R, Chen Y, Li H-Q, Li R-M, Yuan Q-X, et al. Visualising liver fibrosis by phase-contrast $\mathrm{X}$-ray imaging in common bile duct ligated mice. Eur Radiol. 2013;23(2):417-23.

40. Duan J, Hu C, Luo S, Zhao X, Wang T. Microcomputed tomography with diffraction-enhanced imaging for morphologic characterization and quantitative evaluation of microvessel of hepatic fibrosis in rats. PLoS One. 2013;8(10):e78176.

41. • Hu D-d, Chen Y, Bihi A, Li X-m, Wang T-l, Wang B-e et al. A new conversation between radiology and pathology-identifying microvascular architecture in stages of cirrhosis via diffraction enhanced imaging in vitro. PloS One. 2014;9(2):e87957. In this study, Hu et al., show that both cirrhosis and fibrosis of the liver can be measured and stratefied with DEI.

42. Schwab F, Schleede S, Hahn D, Bech M, Herzen J, Auweter S, et al. Comparison of contrast-to-noise ratios of transmission and dark-field signal in grating-based X-ray imaging for healthy murine lung tissue. Zeitschrift für Medizinische Physik. 2013;23(3):236-42.

43. Schleede S, Meinel FG, Bech M, Herzen J, Achterhold K, Potdevin G, et al. Emphysema diagnosis using X-ray dark-field imaging at a laser-driven compact synchrotron light source. Proc Natl Acad Sci. 2012;109(44):17880-5.

44. - Meinel FG, Schwab F, Schleede S, Bech M, Herzen J, Achterhold $\mathrm{K}$ et al. Diagnosing and mapping pulmonary emphysema on X-ray projection images: incremental value of grating-based X-ray dark-field imaging. PloS One. 2013;8(3):e59526. In this study, Meinel et al., demonstrate the effectiveness of using PCI for stratefying the degree of emphysema in a lung.

45. Connor DM, Cole EB, Zhong Z, Parham CA, Pisano ED. Preliminary performance measurements from a second generation diffraction enhanced imaging system. Proceedings of SPIE. 2012;8313(Conference abstract):Paper 8313-163.

46. Ritman EL. X-ray phase-based imaging: the third wave. Acad Radiol. 2009;16(8):909-10. doi:10.1016/j.acra.2009.06.003. 